\title{
Oscillations During Thermonuclear X-ray Bursts
}

\author{
Tod E. Strohmayer ${ }^{1}$ \\ ${ }^{1}$ NASA's Goddard Space Flight Center, Mail Code 662, Greenbelt, MD 207r1, USA
}

\begin{abstract}
High amplitude, nearly coherent X-ray brightness oscillations during thermonuclear X-ray bursts were discovered with the Rossi X-ray Timing Explorer (RXTE) in early 1996. Spectral and timing evidence strongly supports the conclusion that these oscillations are caused by rotational modulation of the burst emission and that they reveal the spin frequency of neutron stars in low mass X-ray binaries, a long sought goal of X-ray astronomy. Studies carried out over the past year have led to the discovery of burst oscillations in four new sources, bringing to ten the number with confirmed burst oscillations. I review the status of our knowledge of these oscillations and indicate how they can be used to probe the physics of neutron stars. For a few burst oscillation sources it has been proposed that the strongest and most ubiquitous frequency is actually the first overtone of the spin frequency and hence that two nearly antipodal hot spots are present on the neutron star. This inference has important implications for both the physics of thermonuclear burning as well as the mass - radius relation for neutron stars, so its confirmation is crucial. I discuss recent attempts to confirm this hypothesis for $4 \mathrm{U}$ 1636-53, the source for which a signal at the putative fundamental (290 $\mathrm{Hz}$ ) has been claimed.
\end{abstract}

\section{INTRODUCTION}

Since its launch in December, 1995 NASA's Rossi X-ray Timing Explorer has provided astronomers with a fundamental new view of neutron stars, and in particular those which are accreting in binary systems. Since the discovery of rapidly rotating neutron stars as millisecond radio pulsars it has been suspected that neutron stars in low mass X-ray binaries (LMXB) are spun up to millisecond periods by the capture of angular momentum via mass transfer from an accretion disk. Efforts to confirm this hypothesis by detecting millisecond X-ray pulsars in LMXBs went unrewarded for many years. This situation changed dramatically with the advent of RXTE. Within a few months of its launch RXTE observations had provided strong evidence suggesting that neutron stars in LMXB are spinning with frequencies $\geq 300 \mathrm{~Hz}$. These first indications came with the discovery of high frequency (millisecond) X-ray brightness oscillations, "burst oscillations," during thermonuclear (Type I) X-ray bursts from several neutron star LMXB systems (see Strohmayer et al. 1996; Smith, Morgan \& Bradt 1997; Zhang et al. 1996).

As of this writing burst oscillation detections have been claimed for a total of ten different LMXB systems, with four of these only appearing in the past few months (see Strohmayer et al. 1996; Zhang et al. 1996; Smith, Morgan \& Bradt 1997; Strohmayer et al. 1997; Markwardt, Strohmayer, \& Swank 1998; Zhang et al. 1998; Wijnands, Strohmayer \& Franco 2000; Galloway et al. 2000; Chakrabarty 2000; Heise 2000). The (1) observed amplitudes, (2) high coherence, and (3) long-term stability of these oscillations provide overwhelming evidence that they are produced by spin modulation of the X-ray burst flux coming from the neutron star surface. Moreover, there properties are dramatically in contradiction to what would be expected from, e.g., models where the modulations reside in the accretion disk surrounding the neutron star.

The observed frequencies are in the range from $\approx 270-620 \mathrm{~Hz}$, bearing a strong similarity to the observed frequency distribution of binary millisecond radio pulsars (Taylor, Manchester \& Lyne 1993; Bildsten 2000) and consistent with some theoretical determinations of spin periods which can be reached via accretioninduced spin-up (Webbink, Rappaport \& Savonije 1983). Very recently Heise et al. (2000) reported evidence of a burst oscillation from the only known accreting millisecond pulsar SAX J1808-369 at a frequency 
consistent with its precisely known spin frequency of $401 \mathrm{~Hz}$ (Wijnands \& van der Klis 1998; Chakrabarty \& Morgan 1998). This provides additional compelling evidence that the burst oscillations are produced by spin modulation of the X-ray burst flux.

In this contribution I will briefly review our observational understanding of these oscillations, with emphasis on how they can be understood in the context of spin modulation of the X-ray burst flux. As this field is evolving rapidly what I present will necessarily be a snapshot of our current understanding. I will discuss how detailed modelling of the oscillations can be used to place interesting constraints on the masses and radii of neutron stars and therefore the equation of state (EOS) of supranuclear density matter. Inferences which can be drawn regarding the physics of thermonuclear burning will also be discussed. I will show that the physical inferences which can be drawn in these areas depend crucially on whether one or two (anitpodal) hot spots produce the observed modulations. I will present an analysis to try and confirm the two spot scenario for $4 \mathrm{U}$ 1636-53, the source for which the most compelling case for two spots has been made (Miller 1999). I conclude with some outstanding theoretical questions and uncertainties and where future observations and theoretical work may lead.

\section{THEORETICAL EXPECTATIONS}

The notion that X-ray bursts are casued by thermonuclear instabilities in the accreted layer on the surface of a neutron star is now universally accepted. There is no doubt that interesting puzzles remain and our detailed understanding is incomplete, but the basic model is firmly established. The thermonuclear instability which triggers an X-ray burst burns in a few seconds the fuel which has been accumulated on the surface over several hours. This makes it very unlikely that the conditions required to trigger the instability will be achieved simultaneously over the entire stellar surface. This notion, first emphasized by Joss (1978), led to the study of lateral propagation of the burning front over the neutron star surface (see Fryxell \& Woosley 1982, Nozakura, Ikeuchi \& Fujimoto 1984, and Bildsten 1995). The short risetimes of thermonuclear X-ray bursts suggest that convection plays an important role in the physics of the burning front propagation, especially in the low accretion rate regime which leads to large ignition columns (see Bildsten 1998 for a recent review of thermonuclear burning on neutron stars). These studies emphasized that the physics of thermonuclear burning is necessarily a multi-dimensional problem and that localized burning is to be expected, especially at the onset of bursts. As I will describe below, the properties of oscillations near burst onset described above fit nicely with this picture of thermonuclear burning on neutron stars.

\section{PROPERTIES OF BURST OSCILLATIONS}

Burst oscillations with a frequency of $363 \mathrm{~Hz}$ were first discovered from the LMXB 4U 1728-34 by Strohmayer et al. (1996). Since then oscillations in an additional nine sources have been reported. The sources and their observed frequencies are given in Table 1. In the remainder of this section I will briefly review the important observational properties of these oscillations and lay out the evidence supporting spin modulation as the mechanism.

\section{Oscillations at Burst Onset}

Some bursts show very strong oscillations during the $\approx 1-2$ s rises typical of thermonuclear bursts. Strohmayer, Zhang \& Swank (1997) showed that some bursts from 4U 1728-34 have oscillation amplitudes as large as $43 \%$ within $0.1 \mathrm{~s}$ of the observed onset of the burst. They also showed that the oscillation amplitude decreased monotonically as the burst flux increased during the rising portion of the burst lightcurve. Strohmayer et al (1998a) reported on strong pulsations in $4 \mathrm{U} 1636-53$ at $580 \mathrm{~Hz}$ with an amplitude of $\approx 75 \%$ only $\sim 0.1 \mathrm{~s}$ after detection of burst onset. In both of these studies the quoted amplitude is defined as half the peak to peak intensity modulation divided by the mean intensity. The mean level is determined by first subtracting the pre-burst intensity. The presence of modulations of the thermal burst flux approaching $100 \%$ right at burst onset fits nicely with the idea that early in the burst there exists a localized hot spot which is then modulated by the spin of the neutron star. In this scenario the largest modulation amplitudes are produced when the spot is smallest, as the spot grows to encompass more of the neutron star surface, the amplitude drops, consistent with the observations. X-ray spectroscopy during burst rise also supports the inference that X-ray emission is localized on the neutron star near the onset of bursts. Strohmayer, Zhang, \& Swank (1997) found that during burst rise the flux is underluminous compared with intervals later in the 
Table 1. Burst Oscillations Sources and Properties

\begin{tabular}{cccc} 
Sources & Frequency $(\mathrm{Hz})$ & $\Delta \nu(\mathrm{kHz}$ QPO, in Hz $)$ & References $^{1}$ \\
\hline $4 \mathrm{U} 1728-34$ & 363 & $363-280$ & $1,2,3,4,5,13,14$ \\
$4 \mathrm{U} 1636-53$ & 290,580 & 251 & 6,7 \\
$4 \mathrm{U} 1702-429$ & 330 & $315-344$ & 4,9 \\
KS 1731-260 & 524 & 260 & $10,11,12$ \\
Galactic Center & 589 & Unknown & 15 \\
Aql X-1 & 549 & Unknown & 16,17 \\
X1658-298 & 567 & Unknown & 18 \\
4U 1916-053 & 270 & $290-348$ & 19,20 \\
4 U 1608-52 & 619 & $225-325$ & 8,21 \\
SAX J1808-369 & 401 & Unknown & 22,23 \\
\hline
\end{tabular}

${ }^{1}$ References: (1) Strohmayer et al. (1996); (2) Strohmayer, Zhang, \& Swank (1997); (3) Mendez \& van der Klis (1999); (4) Strohmayer \& Markwardt (1999); (5) Strohmayer et al. (1998b); (6) Strohmayer et al. (1998a); (7) Miller (1999); (8) Mendez et al. (1998); (9) Markwardt, Strohmayer \& Swank (1999) (10) Smith, Morgan, \& Bradt (1997); (11) Wijnands \& van der Klis (1997); (12) Muno et al. (2000); (13) van Straaten et al. (2000); (14) Franco (2000); (15) Strohmayer et al (1997); (16) Zhang et al. (1998); (17) Ford (1999); (18) Wijnands, Strohmayer \& Franco (2000); (19) Boirin et al. (2000); (20) Galloway et al. (2000); (21) Chakrabarty (2000); (22) Heise (2000); (23) Ford (2000)

burst which have the same observed black body temperature, suggesting that during the rise only a portion of the surface of the neutron star is producing the X-ray emission. As the burst progresses the burning area increases in size until the entire surface is involved.

\section{Coherence of Burst Oscillations}

The observed oscillation frequency during a burst is usually not constant. Often the frequency is observed to increase by $\approx 1-3 \mathrm{~Hz}$ in the cooling tail, reaching a plateau or asymptotic limit (see Strohmayer et al. 1998a). This behavior is common to all the burst oscillation sources, and it would appear that the same physical mechanism is involved, however, there have been reports of decreases in the oscillation frequency with time. For example, Strohmayer (1999) and Miller (1999) identified a burst from 4U 1636-53 with a spin down of the oscillations in the decaying tail. This burst also had an unusually long decaying tail which may have been related to a "reheating" episode and could also acount for the spin down. Muno et al. (2000) reported on a burst from KS 1731-260 which also showed an episode where the frequency dropped, however, in this case they found no evidence for unusual flux enhancements or spectral varations during the episode. Strohmayer (1997) suggested that relative motions of the hot spot and/or burning front on the neutron star surface might also introduce both spin up and spin down episodes.

Strohmayer et. al (1997) have argued that the time evolution of the frequency results from angular momentum conservation of the thermonuclear shell. The burst expands the shell, increasing its rotational moment of inertia and slowing its spin rate. Near burst onset the shell is thickest and thus the observed frequency lowest. The shell spins back up as it cools and recouples to the underlying neutron star. Calculations indicate that the $\sim 10 \mathrm{~m}$ thick pre-burst shell expands to $\sim 30 \mathrm{~m}$ during the flash (see Joss 1978; Bildsten 1995; Cumming \& Bildsten 2000), which gives a frequency shift of $\approx 2 \nu_{\text {spin }}(20 \mathrm{~m} / R)$, where $\nu_{\text {spin }}$ and $R$ are the stellar spin frequency and radius, respectively. For the several hundred $\mathrm{Hz}$ spin frequencies inferred from burst oscillations this gives a shift of $\sim 2 \mathrm{~Hz}$, similar to that observed. Strohmayer \& Markwardt (1999) showed that the frequency evolution in 4U 1728-34 and 4U 1702-429 is highly phase coherent. They modelled the frequency drift and showed that a simple exponential "chirp" model of the form $\nu(t)=\nu_{0}\left(1-\delta_{\nu} \exp (-t / \tau)\right)$, works remarkably well, producing quality factors $Q \equiv \nu_{0} / \Delta \nu_{F W H M} \sim 4,000$. Muno et al. (2000) performed a similar analysis on bursts from KS 1731-26 and concluded that the burst oscillations from this source were also phase coherent. These results argue strongly that the mechanism which produces the modulations is intrinsically a highly coherent one. 
Recently, Galloway et al. (2000) reported a $3.5 \mathrm{~Hz}$ frequency shift in a burst from 4U 1916-053 with $272 \mathrm{~Hz}$ oscillations. They suggested that such a large change, $\sim 1.3 \%$ might be inconsistent with expansion of the thermonuclear burning layer because of the magnitude of the implied height change of $\sim 80 \mathrm{~m}$. Wijnands, Strohmayer \& Franco (2000) found a $\sim 5 \mathrm{~Hz}$ frequency shift in a burst from 4U 1658-298 with a $567 \mathrm{~Hz}$ oscillation, which may also be uncomfortably large given current estimate of the expansion of burning layers (Cumming \& Bildsten 2000). Note, however, that the current theoretical estimates do not include the rotational lowering of the effective surface gravity and are also hydrostatic calculations. Dynamic motions of the layer may also contribute to changes in the height of the burning layer. These effects could increase the height of the burning layer and allow for greater frequency drifts than current calculations suggest. Also, a combination of height changes as well as lateral motions with respect to the neutron star surface may be at work simultaneously. Clearly more theoretical work is required to determine if expansion of the burning layer is indeed the primary mechanism responsible for the frequency drifts and also to understand the nature of the less commonly observed spin down episodes.

\section{Long Term Stability}

The accretion-induced rate of change of the neutron star spin frequency in a LMXB is approximately $1.8 \times 10^{-6} \mathrm{~Hz} \mathrm{yr}^{-1}$ for typical neutron star and LMXB parameters. The Doppler shift due to orbital motion of the binary can produce a frequency shift of magnitude $\Delta \nu / \nu=v \sin i / c \approx 2.05 \times 10^{-3}$, again for representative LMXB system parameters. This doppler shift easily dominates over any possible accretioninduced spin change on orbital to several year timescales. Therefore the extent to which the observed burst oscillation frequencies are consistent with possible orbital Doppler shifts, but otherwise stable over $\approx$ year timescales, provides strong support for a highly coherent mechanism which sets the observed frequency.

At present, the best source available to study the long term stability of burst oscillations is $4 \mathrm{U}$ 1728-34. Strohmayer et al. (1998b) compared the observed asymptotic frequencies in the decaying tails of bursts separated in time by $\approx 1.6$ years. They found the burst frequency to be highly stable, with an estimated time scale to change the oscillation period of about 23,000 year. It was also suggested that the stability of the asymptotic periods might be used to infer the X-ray mass function of LMXB by comparing the observed asymptotic period distribution of many bursts and searching for an orbital Doppler shift. The source $4 \mathrm{U}$ 1636-53 is a good candidate for such an effort because its orbital period is known (3.8 hrs). Strohmayer et al. (1998b) compared the highest observed frequencies in three different bursts from 4U 1636-53. The frequencies in these bursts alone were consistent with a typical orbital velocity for the neutron star. However, study of additional bursts reveals a greater range of highest frequencies than can likely be accounted for by orbital motion alone (Giles \& Strohmayer 2001). A possible explanation of this within the context of the spin modulation scenario is that not every burst has relaxed to the asymptotic value before the oscillations fade below the detection level. Nevertheless, the observed distribution of frequencies in 4U 1636-53 does suggest the existence of an upper limit, which can naturally be associated with the spin frequency (Giles \& Strohmayer 2001).

\section{Burst Oscillations and Source State}

Several recent studies have focused on how the presence and properties of burst oscillations may or may not correlate with other properties of these sources, for example, their spectral state and mass accretion rate. Muno et al. (2000) found that bursts from KS 1731-26 with oscillations appear to only occur when the source is on the banana branch in the X-ray color-color diagram. They also found that these bursts were all radius expansion bursts. Cumming \& Bildsten (2000) suggested that such bursts were likely pure Helium flashes and that it would be more likely for these to show oscillations because the radiative diffusion time is short compared to the inferred shearing time of the burning layer, making it more likely that a modulation would survive. Franco (2000) and van Straaten et al. (2000) showed that bursts from 4U 1728-34 with oscillations also occur preferentially on the banana branch, but they did not find a similar relationship with radius expansion as for KS 1731-26. Franco (2000) also found that the strength of oscillations was correlated with position in the color-color diagram. These results are begining to provide new insights into how mass accretion rate effects thermonuclear burning on neutron stars. 


\section{BURST OSCILLATIONS AS PROBES OF NEUTRON STARS}

Precise modelling of burst oscillations holds great promise for providing new insights into a variety of physics questions concerning the structure and evolution of neutron stars. For example, pulse amplitudes and shapes produced by rotational modulation of a hot spot contain information on both the compactness, $M / R$, of the neutron star as well as the rotational velocity of the hot spot. Since the spin frequency is known the velocity is directly proportional to the stellar radius $R$. In addition, the rotational motion provides us with a snapshot view of the propagation of the thermonuclear instability.

\section{M - R Constraints}

One of the crucial bits of physics that makes compactness constraints possible is the bending of photon trajectories in a strong gravitational field. The strength of such deflection is a function of the stellar compactness, $G M / c^{2} R$, with more compact stars producing greater deflections and therefore weaker spin modulations. An upper limit on the compactness can be set since a star more compact than this limit would not be able to produce a modulation as large as observed. Complementary information comes from the pulse shape, which can be inferred from the strength of harmonics. Information on both the amplitude and harmonic content can thus be used to bound the compactness.

Stellar rotation also plays a role in the observed properties of spin modulation pulsations. For example, a $10 \mathrm{~km}$ radius neutron star spinning at $400 \mathrm{~Hz}$ has a surface velocity of $v_{\text {spin }} / c \leq 2 \pi \nu_{\text {spin }} R / c \approx 0.084$ at the rotational equator. This motion produces a relativistic abberation as well as a Doppler shift of magnitude $\Delta E / E \approx v_{\text {spin }} / c$ (see Chen \& Shaham 1989). Measurement of the pulse phase dependent Doppler shift in the X-ray spectrum would provide additional evidence supporting the spin modulation model and also provides one of the few direct methods to infer the radius of a neutron star. Ford (1999) has analysed data during a burst from Aql X-1 and finds that the softer photons lag higher energy photons in a manner which is qualitatively similar to that expected from a rotating hot spot.

Detailed modelling of pulse shapes and pulse phase spectra can then be used to determine a confidence region in the mass - radius plane for neutron stars. Miller \& Lamb (1998) have investigated the amplitude of rotational modulation pulsations as well as harmonic content assuming emission from a point-like hot spot. They also showed that knowledge of the angular and spectral dependence of the emissivity from the neutron star surface can have important consequences for the derived constraints. Recently, Nath, Strohmayer \& Swank (2000) have used a rotating hot spot model to fit bolometric pulse profiles observed on the rising edge of bursts from $4 \mathrm{U}$ 1636-53. They find that the inferred constraints depend very sensitively on whether or not two spots are present. Much more restrictive compactness constraints can be achieved if the two spot hypothesis is correct. The main reason for this being that large amplitudes are much more difficult to achieve with two spots than one. The large observed amplitudes from $4 \mathrm{U}$ 1636-53 require a large neutron star which in turn requires a very stiff EOS. The inferred radius is also sensitive to this two spot assumption, since uncertainty in the number of spots gives a factor of two ambiguity in the rotational velocity.

\section{Physics of Nuclear Burning and Propagation}

The properties of burst oscillations can tell us a great deal about the processes of nuclear burning on neutron stars. The amplitude evolution during the rising phase of bursts contains information on how rapidly the flame front is propagating. If the anitpodal spot hypothesis to explain the presence of a subharmonic in $4 \mathrm{U}$ 1636-53 is correct, then it has important implications for the propagation of the instability from one pole to another in $\approx 0.2 \mathrm{~s}$ (see Miller 1999). In addition, a two pole flux anistropy suggests that the nuclear fuel is likely pooled by some mechanism, perhaps associated with the magnetic field of the star. In order to have a robust understanding of the implications of burst oscillations for neutron star structure and thermonuclear propagation it is essential to remove any ambiguity concerning the number of hot spots producing the observed modulations.

\section{U 1636-53: WHAT IS THE SPIN FREQUENCY?}

Zhang et al. (1996) reported the discovery of $580 \mathrm{~Hz}$ oscillations in bursts from 4U 1636-53. Observations of the accretion driven flux from this source yielded a pair of kilo-Hertz quasiperiodic oscillations (QPO) with a frequency separation of about $280 \mathrm{~Hz}$ (see Mendez et al. 1998). The beat frequency interpretation for the twin $\mathrm{kHz}$ peaks suggests that the observed frequency separation of the $\mathrm{kHz}$ QPO peaks should be 
Table 2. Bursts from 4U 1636-53 used in the subharmonic search

\begin{tabular}{cccccc} 
Segment & Burst & Obs. ID. & Date & HJD - 2450000 & $Z_{1}^{2}$ \\
\hline 1 & 1 & $30053-02-02-02$ & $8 / 19 / 98$ & 1044.991053 & 124 \\
2 & 2 & $30053-02-01-02$ & $8 / 20 / 98$ & 1045.654542 & 54 \\
3 & 3 & $30053-02-02-00$ & $8 / 20 / 98$ & 1045.719849 & 148 \\
4 & 4 & $40028-01-02-00$ & $2 / 27 / 99$ & 1236.865609 & $61^{a}$ \\
5 & 4 & $40028-01-02-00$ & $2 / 27 / 99$ & 1236.865609 & $52^{b}$ \\
6 & 5 & $40028-01-08-00$ & $6 / 18 / 99$ & 1348.493173 & 111 \\
7 & 6 & $40028-01-10-00$ & $9 / 25 / 99$ & 1447.360320 & 69 \\
\hline
\end{tabular}

${ }^{1}$ Heliocentric Julian Date

close to the spin frequency of the neutron star (see Strohmayer et al. 1996; Miller, Lamb \& Psaltis 1998). The observed $\sim 280 \mathrm{~Hz} \mathrm{kHz}$ QPO separation in 4U 1636-53 prompted Miller (1999) to search for a $\sim 290$ $\mathrm{Hz}$ subharmonic of the $580 \mathrm{~Hz}$ signal during bursts from this source. Miller (1999) added the signals from 5 different bursts and reported a detection of the subharmonic with a chance detection probability of $4 \times 10^{-5}$ and suggested that $290 \mathrm{~Hz}$ is the neutron star spin frequency.

\section{Coherent Addition of Signals from Different Bursts}

Miller (1999) used a "matched waveform filtering" or "template filtering" technique to search for the subharmonic of the already detected $580 \mathrm{~Hz}$ signal in bursts from $4 \mathrm{U}$ 1636-53. He did this by using the observed signal at $580 \mathrm{~Hz}$ to predict what the $290 \mathrm{~Hz}$ signal would be and to then use that as the template in his search. Strohmayer \& Markwardt (1999) have investigated the phase and frequency evolution of pulsations in bursts using the $Z_{n}^{2}$ statistic,

$$
Z_{n}^{2}=2 / N \sum_{k=1}^{n}\left(\sum_{j=1}^{N} \cos \left(k \phi_{j}\right)\right)^{2}+\left(\sum_{j=1}^{N} \sin \left(k \phi_{j}\right)\right)^{2},
$$

where $N$ is the total number of photons in the time series, $\phi_{j}$ are the phases of each photon derived from a frequency model, $\nu(t)$, vis. $\phi_{j}=2 \pi \int_{0}^{t_{j}} \nu\left(t^{\prime}\right) d t^{\prime}$, and $n$ is the total number of harmonics added together. Here I use this method to search for harmonics and the subharmonic of the $580 \mathrm{~Hz}$ signal from $4 \mathrm{U}$ 1636-53 in a different set of bursts than studied by Miller (1999).

For the burst oscillations, which are highly sinusoidal, it is appropriate to set $n=1$. The $Z_{1}^{2}$ statistic is particularly well suited to event mode data, since no binning is introduced, and it has the same statistical properties as the well known Leahy normalized power spectrum, which for a Poisson process is distributed as the $\chi^{2}$ function with 2 degrees of freedom. All of the bursts discussed here were observed with the Proportional Counter Array (PCA) onboard RXTE and sampled with $125 \mu \mathrm{s}$ (1/8192 s) resolution. Following Miller (1999) I search for the subharmonic during pulsations on the rising edge of bursts. Since I am trying to confirm a previous detection I use a different sample of bursts than were analysed by Miller (1999). After compiling a catalog of all available bursts from $4 \mathrm{U}$ 1636-53 I searched for those with significant oscillations on the rising edge and found six suitable for the search. The bursts I used are summarized in Table 2.

\section{Burst Oscillations in the Complex Plane}

The $\sin \phi$ and $\cos \phi$ terms in the $Z_{1}^{2}$ function can be thought of as the real and imaginary components of a complex expression, $Z=\sum_{j}\left(\cos \phi_{j}+i \sin \phi_{j}\right)$. $Z_{1}^{2}$ is just the summed squared modulus of this expression. Graphically we can represent the evolution of oscillations in a given data segment by plotting the positions in the complex plane of the set of cumulative $Z \mathrm{~s}$ for each segment, with each point being obtained by adding in the next event in the sum. In such a representation, a coherent signal will be evident as a straight line in the complex plane with a position angle which represents the phase of the oscillations. The length squared of such a line is just the peak $Z_{1}^{2}$ for that set of events. 

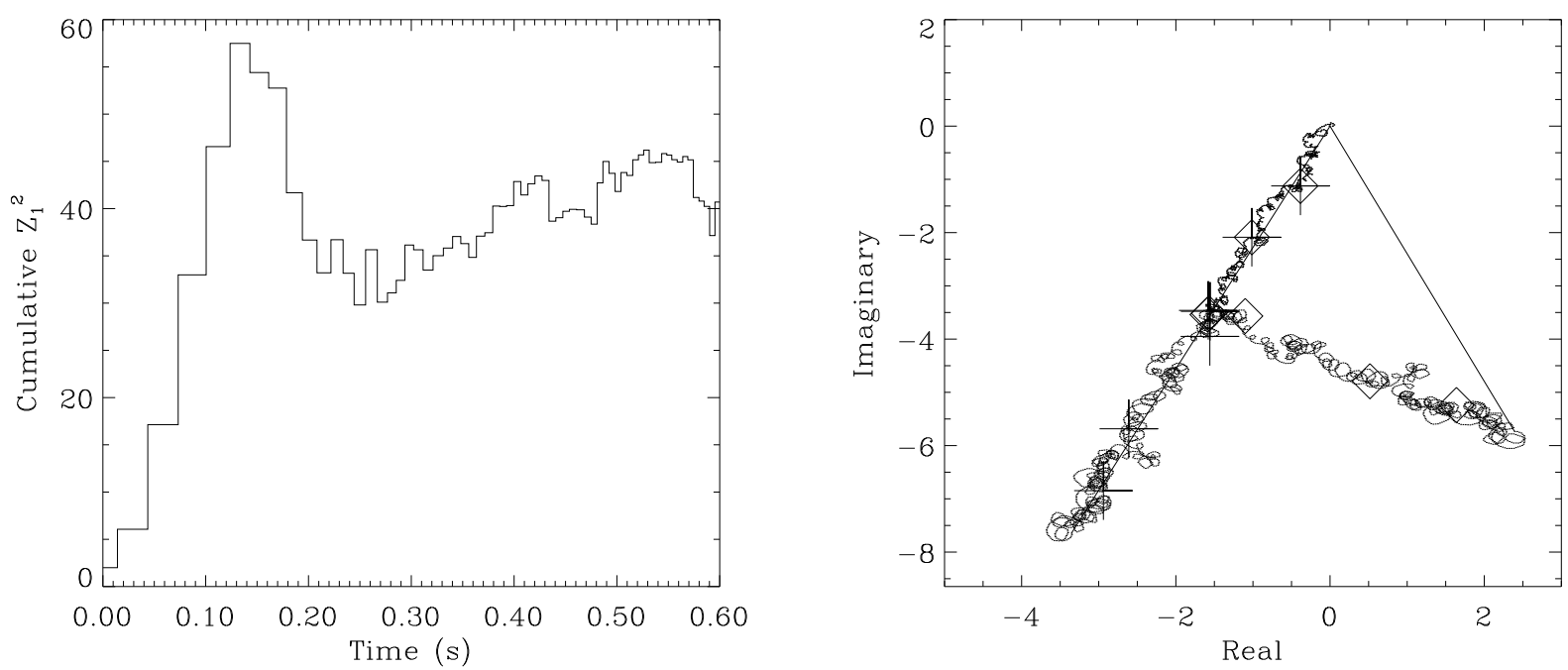

Fig. 1. Cumulative $Z_{1}^{2}$ for burst 4 using a constant frequency of $580.8 \mathrm{~Hz}$ (left panel). There is a very strong oscillation in the first $0.1 \mathrm{~s}$ of the burst followed by a fading of the oscillation (evidenced by the drop in $Z_{1}^{2}$ between 0.15 and $0.25 \mathrm{~s}$ ). The signal strengthens again, but there is a phase offset compared with an extrapolation of the best frequency inferred from the first $0.15 \mathrm{~s}$ of data. The right panel shows the phase evolution in the complex plane using the best frequency for the interval from 0 to $0.15 \mathrm{~s}$. The phase evolution is shown both with (crosses) and without (diamonds) a phase shift in the 2 nd data segment of $\sim 0.234$ of a cycle. Including the phase shift straightens out the bend in the phase evolution. The symbols are plotted every $0.05 \mathrm{~s}$.

Evidence for a Phase Jump

For each burst in our sample I used frequency models, $\nu(t)$, with both a constant and a linearly evolving frequency. I found no significant evidence for a changing frequency with one exception. Burst 4 showed an unusual phase evolution when the cumulative phases for the best constant frequency model were plotted in the complex plane. This behavior is illustrated in Figure 1. This burst has a very strong oscillation begining $\leq 0.05 \mathrm{~s}$ after onset (see Figure 1, left panel). The oscillation fades for about $0.1 \mathrm{~s}$, evidenced by the drop in $Z_{1}^{2}$, before strengthening again, however, when the oscillation is seen again there is an apparent phase offset compared to what would be expected from the best frequency inferred from the first $0.15 \mathrm{~s}$ of the burst. The phase offset is about $1 / 4$ of a cycle and it was accumulated over $\sim 0.1 \mathrm{~s}$ (see Figure 1, right panel), implying a limit on $d P / d t \sim 4 \times 10^{-3} \mathrm{~s} \mathrm{~s}^{-1}$ during this time. Because of this phase evolution, for the purposes of conducting the subharmonic search burst 4 was broken up into two separate segments. Each segment was modelled separately using a constant frequency model.

\section{$\underline{\text { Subharmonic Search }}$}

The search procedure had the following steps. 1) I first extracted a set of X-ray events from the start of each burst, $t_{0}$ to the peak, $t_{\text {peak }}$. 2) I then maximized the $Z_{1}^{2}$ signal at $580 \mathrm{~Hz}$ separately for each burst. I did this by finding the event time $t_{\text {max }}^{i}$ between $t_{0}^{i}$ and $t_{\text {peak }}^{i}$ which gives the maximum $Z_{1}^{2}$. I found, with one exception, that for the rising intervals a constant frequency model was adequate. That is, I did not find that a linear time dependence (either increasing or decreasing) of the frequency improved $Z_{1}^{2}$ significantly, except in the one case noted above. Therefore for each data segment I determined the constant frequency $\nu^{i}$ which gave the maximum $Z_{1}^{2}$ for each burst. I then used the events and the frequency, $\nu^{i}$, to compute a set of phases, $\phi_{j}^{i}=2 \pi \nu^{i} t_{j}$, for each burst, where the $i$ subscript identifies the different bursts. 3) I next combined the phases from each burst to determine a maximum $Z_{1}^{2}$. This was done by first determining a phase offset, $\delta \phi_{i}$, for each burst which maximized the $Z_{1}^{2}$ signal computed using events from that burst and the first burst, whose phase was used as an initial reference without loss of generality. This amounted to 


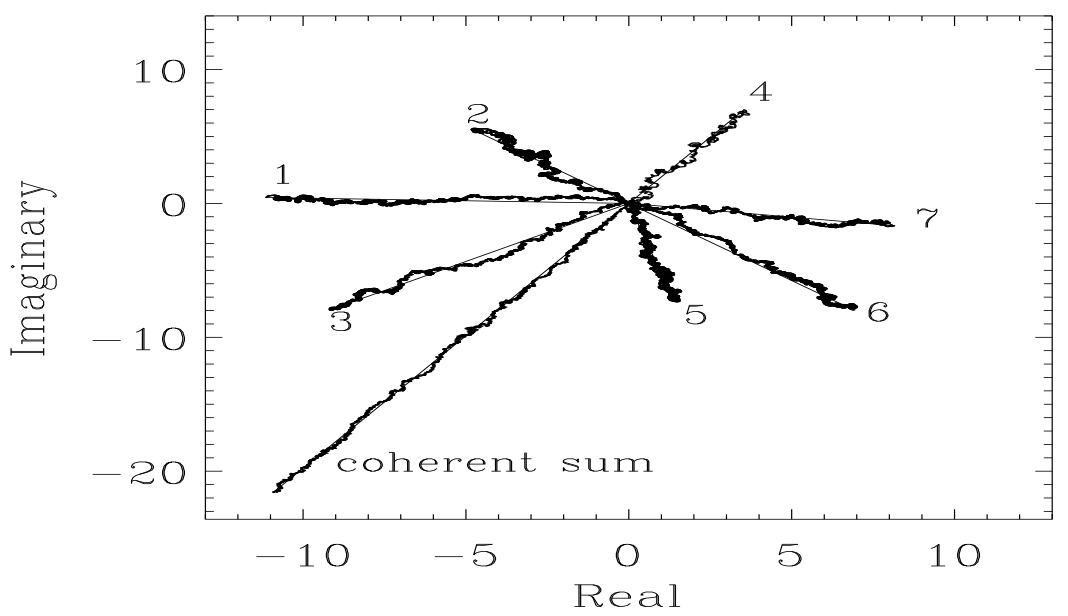

Fig. 2. Cumulative phases in the complex plane using the constant frequency model for each data segment used in our analysis. The coherent sum of all 7 intervals is also shown. The fact that each segment can be approximated by a straight line, to within statistical precision, is a demonstration that each segment is phase coherent. The total $Z_{\text {sum }}^{2}$ at $580 \mathrm{~Hz}$ is $\sim 585$. This can be compared with a signal of $\sim 138$ obtained by Miller (1999) using 5 different bursts. For clarity an arbitrary phase offset has been added to some of the segments so they do not overlap.

computing the following;

$$
Z_{\text {sum }}^{2}=\frac{2}{\sum_{i=1}^{m_{\text {burst }}} N_{i}}\left[\left(\sum_{i=1}^{m_{\text {burst }}} \sum_{j=1}^{N_{i}} \cos \left(\phi_{j}^{i}+\delta \phi^{i}\right)\right)^{2}+\left(\sum_{i=1}^{m_{\text {burst }}} \sum_{j=1}^{N_{i}} \sin \left(\phi_{j}^{i}+\delta \phi^{i}\right)\right)^{2}\right],
$$

where $N_{i}$ is the number of X-ray events in burst $i$, and $m_{\text {burst }}$ is the number of different bursts added together. The $\delta \phi$ are the phase shifts which align each burst with respect to burst 1 (ie. $\delta \phi_{1} \equiv 0$ ).

I carried out this procedure for the 7 data segments in Table 1. The results are summarized in Figure 2 where I have plotted the cumulative phases for each data segment as well as the total coherent sum. The segments are numbered according to the label in Table 2. The fact that each line segment is straight to within statistical precision attests to its phase coherence. The total $Z_{\text {sum }}^{2}$ power at $580 \mathrm{~Hz}$ of $\sim 585$ can be compared to a power of $\sim 138$ quoted by Miller (1999) for the coherent sum of the 5 different bursts he analysed.

Having coherently added all the data segments I can now conduct harmonic and subharmonic searches. A harmonic search is straightforward. I simply evaluated $Z_{\text {sum }}^{2}\left(k \phi_{j}^{i}\right)$ with $k=2,3,4 \ldots$ etc. For any $k$ the $Z_{\text {sum }}^{2}$ will be distributed as $\chi^{2}$ with 2 degrees of freedom, so that I can determine a probability that a harmonic signal is present by computing the probability of drawing $Z_{\text {sum }}^{2}\left(k \phi_{j}^{i}\right)$ from the $\chi^{2}$ distribution. Evaluating $Z_{\text {sum }}^{2}\left(k \phi_{j}^{i}\right)$ for $k=1,2,3,4,5$ did not give a significant detection of any harmonic. Indeed the pulsations are highly sinusoidal, with a maximum power at the 1 st harmonic $(k=2)$ of only 2.81 . This means that any signal at the first harmonic is at least 15 times weaker than the $580 \mathrm{~Hz}$ signal.

To search for the subharmonic is a little more difficult because there is a $\pi$ phase ambiguity between the crests of any putative $290 \mathrm{~Hz}$ oscillation when combining two different bursts (see Miller 1999). I simply multiplied each set of phases by $1 / 2$ to search the first subharmonic, $590 / 2 \mathrm{~Hz}$, however, to combine 7 separate segments I must allow for a total of $2^{6}=64$ different combinations of the phases. This is equivalent to a search with 64 independent trials. The results of this search are shown in Figure 3. I did not detect the subharmonic. I found a peak power at the subharmonic of only $\sim 10$, which means that the signal amplitude at $290 \mathrm{~Hz}$ is less than $\sim 13 \%$ of the amplitude at $580 \mathrm{~Hz}$. If the subharmonic signal would have been at the same strength as quoted by Miller (1999) I would have found a power at the subharmonic of 

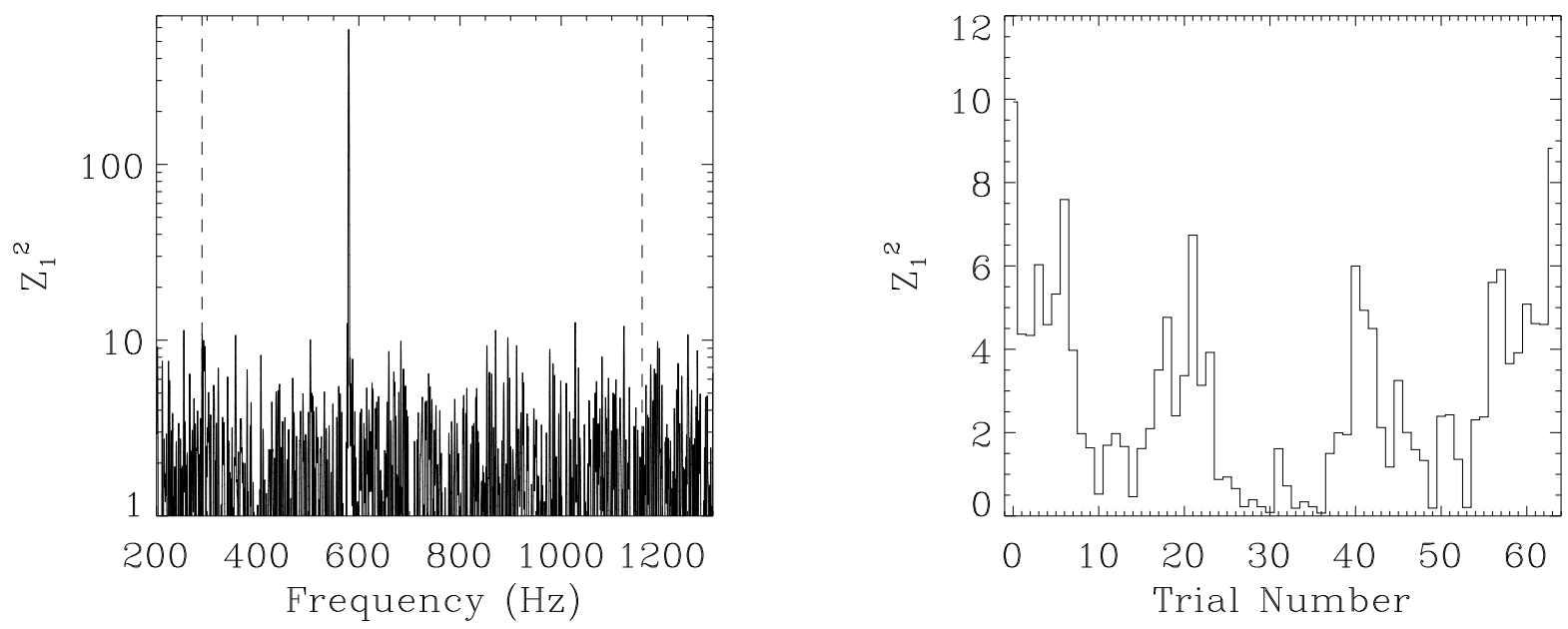

Fig. 3. Coherent $Z_{\text {sum }}^{2}$ for the 7 burst intervals (left). Note the logarithmic y-axis scale. The strong peak at 580 $\mathrm{Hz}$ has a power of $\sim 585$. The vertical dashed lines mark the positions of the first subharmonic $(290 \mathrm{~Hz})$ and the first harmonic $(1160 \mathrm{~Hz})$, neither of which are detected. The right panel shows $Z_{\text {sum }}^{2}$ for the 64 possible phase permutations of the first subharmonic when combining 7 independent segments, the largest power was $\sim 9.98$ and with 64 trials is not significant.

$\sim 110$ which would have been easily detected in these data. Analysis of these six bursts using the waveform matching technique of Miller (1999) confirms the lack of a significant signal at $290 \mathrm{~Hz}$ (C. Miller, personal communication).

\section{SUMMARY AND CONCLUSIONS}

As outline above the inferences which can be made concerning neutron star structure and thermonuclear burning depend importantly on whether one or two hot spots produce the observed burst oscillations. So the question remains, what is the spin frequency of $4 \mathrm{U} 1636-53$, is it $290 \mathrm{~Hz}$ or $580 \mathrm{~Hz}$ ? Based on the work presented here I am not able to confirm the $290 \mathrm{~Hz}$ interpretation presented by Miller (1999). In a further effort to try and confirm the subharmonic detection reported by Miller (1999), I used the $Z_{1}^{2}$ method outlined here to investigate the same set of bursts analysed by Miller (1999). I was able to coherently add the signals at $580 \mathrm{~Hz}$, obtaining a peak $Z_{1}^{2}=135$, a value very close to that found by Miller (1999) using the "matched waveform filtering" method, however, I did not find a significant peak at the $290 \mathrm{~Hz}$ subharmonic. I found a $Z_{1}^{2}$ of only $\sim 6.8$ which given 16 trials is not significant. Note, however, that an analysis using the $Z_{1}^{2}$ technique discussed here but applied to the same data intervals used by Miller (1999) confirms the significance of the signal at $290 \mathrm{~Hz}$ reported by Miller (C. Markwardt, personal communication). It seems likely that the different data intervals selected by the two methods accounts for the different results. This conclusion is further supported by the fact that I was able to increase the measured power at the $290 \mathrm{~Hz}$ subharmonic by including a larger interval from one of the bursts in the sample originally studied by Miller (1999) (ocurring on December 28, 1996 at 23:47:25 UTC: obsid 10088-01-07-02). In fact, it appears that the detection reported by Miller (1999), and confirmed by Markwardt (2001), is dependent strongly on this one burst. Moreover, the inability to detect a subharmonic in subsequent bursts reported here supports the notion that perhaps only a small fraction of bursts produce significant oscillations at $290 \mathrm{~Hz}$. The results described here should not be seen as an attempt to discredit or disparage the previous work by Miller (1999), rather, it is simply a matter of a result with very important implications requiring confirmation, especially in an independent data set.

The attempt to detect the subharmonic in new data from $4 \mathrm{U}$ 1636-53 has led to several interesting puzzles, the first of which is, why is the subharmonic apparently so weak most of the time? If two hot spots are 
indeed present, then the lack of a detection places a limit on the flux asymmetry from the two poles as well as on how far from antipodal the spots may be. These new data suggest that the spots must be quite uniform, with the flux asymmetry being less than $\sim 13 \%$. With more detailed modelling it will be possible to convert the limit on the subharmonic amplitude into a constraint on how far apart the spots have to be. If magnetic pooling is at work, then this could give a clue as to the magnetic field geometry on the neutron star. The lack of any harmonic structure is also very important. Both the stellar compactness (if small enough) and the relativistic rotational motion of the hot spot should introduce harmonic structure into the pulse shape. The lack of harmonic structure suggests that the star cannot be bigger than some limit. As the compactness decreases the pulse becomes sharper and also more asymmetric because of the rotational aberration, thus detailed modelling and the limits derived here on the harmonic strength should lead to a lower bound on the compactness.

The other intriguing result concerns the nature of the phase shift indicated for burst 4. Although only one burst in this sample showed evidence for a phase shift there are indications in other bursts from $4 \mathrm{U}$ 1636-53 of similar behavior (Markwardt 2001). So far the putative spin down which is postulated to occur because of the hydrostatic expansion of the thermonuclear burning layer has not been definitively seen. This may be due to the fact that the expansion is expected to happen on a timescale shorter than the radiative diffusion time so that the shell has already puffed up before the photons have a chance to leak out and inform the world of the spin down. The phase shift in burst 4 can be explained by a spin down during the interval when the oscillations fade, and this might conceivably be evidence for the expansion induced shift, but naively one might expect that a more or less smooth spindown would occur, which is not what is seen. Since oscillations on the rising edge of bursts typically fade as the flux increases, it is likely that either the spot or spots are spreading during this time which will reduce the observed amplitude. The nature of this spreading is not well understood. It may be that it could introduce a phase offset when a frequency derived during a time interval when spreading is significant is extrapolated to later times when the spreading has stopped.

\section{ACKNOWLEDGEMENTS}

I would like to thank all the organizers of the COSPAR symposium on black holes and neutron stars, and especially Andrzej Zdziarski, for putting together such a stimulating symposium and for being such gracious hosts. This work benefitted greatly from discussions with Craig Markwardt, Jean Swank, Nitya Nath and Deepto Chakrabarty. I thank the referee, Cole Miller, for his insightful and helpful comments.

\section{REFERENCES}

Bildsten, L., Propagation of Nuclear Burning Fronts on Accreting Neutron Stars: X-ray Bursts and Subhertz Noise, ApJ, 438, 852-875, 1995

Bildsten, L., Thermonuclear Burning on Rapidly Accreting Neutron Stars, in The Many Faces of Neutron Stars, ed. R. Buccheri, A. Alpar \& J. van Paradijs (Dordrecht: Kluwer), 419-460, 1998

Bildsten, L., Gravity Wave Astrophysics and Connections with X-ray Timing, Talk presented at AAS HEAD meeting, Honolulu, HI, 2000

Boirin, L., D. Barret, J. F. Olive, P. F. Bloser, \& J. E. Grindlay, Low and high frequency quasi-periodic oscillations in 4U1915-05, A\& A, 361, 121-138, 2000

Chakrabarty, D. \& E. H. Morgan, The Two-hour Orbit of a Binary Millisecond X-ray Pulsar, Nature, 394, 346-348, 1998

Chakrabarty, D., Millisecond Oscillations and Related Sidebands During Type I X-Ray Bursts, Talk presented at AAS HEAD meeting, Honolulu, HI, 2000

Chen, K. \& J. Shaham, Pulse sharpness and asymmetry in millisecond pulsars, ApJ, 339, 279-290, 1989

Cumming, A. \& L. Bildsten, Rotational Evolution during Type I X-Ray Bursts, ApJ, 544, 453-474, 2000

Ford, E. C., Lag of Low-Energy Photons in an X-Ray Burst Oscillation: Doppler Delays, ApJ, 519, L73-L75, 1999

Ford, E. C., Relativistic Effects in the Pulse Profile of the 2.5 Millisecond X-Ray Pulsar SAX J1808.4-3658, ApJ, 535, L119-L122, 2000

Franco, L., The Effect of the Mass Accretion Rate on the Burst Oscillations in 4U 1728-34, ApJ, submitted, (astro-ph/0009189), 2000 
Fryxell, B. A., \& S. E. Woosley, Finite Propagation Time in Multidimensional Thermonuclear Runaways, ApJ, 261, 332-336, 1982

Galloway, D. K., D. Chakrabarty, M. P. Muno, \& S. Pavlin, Discovery of a $270 \mathrm{~Hz}$ X-Ray Burst Oscillation in the X-Ray Dipper 4U 1916-053, ApJ, submitted, (astro-ph/0010072), 2000

Giles, A. B. \& T. E. Strohmayer, The Relationship Between Doppler Shifted X-ray QPOs and the Binary Optical Phase of $4 \mathrm{U} 1636-53$, in preparation, 2001

Heise, J., The transient X-ray sky: highlights from the BeppoSAX Wide Field Cameras, Talk presented at AAS HEAD meeting, Honolulu, HI, 2000

Joss, P. C., Helium-burning flashes on an accreting neutron star - A model for X-ray burst sources, $A p J, 225$, L123-L127, 1978

Markwardt, C. B., T. E. Strohmayer, \& J. H. Swank, Observation of Kilohertz Quasi-periodic Oscillations from the Atoll Source 4U 1702-429 by the Rossi X-Ray Timing Explorer, ApJ, 512, L125-L129, 1999

Markwardt, C. B. personal communication, 2001

Mendez, M., M. van der Klis, \& J. van Paradijs, Difference Frequency of Kilohertz QPOs Not Equal to Half the Burst Oscillation Frequency in 4U 1636-53, ApJ, 506, L117-L119, 1998

Mendez, M. \& M. van der Klis, Precise Measurements of the Kilohertz Quasi-periodic Oscillations in 4U 1728-34, ApJ, 517, L51-L54, 1999

Miller, M. C. \& F. K. Lamb, Bounds on the Compactness of Neutron Stars from Brightness Oscillations during X-Ray Bursts, ApJ, 499, L37-L40, 1998

Miller, M. C., F. K. Lamb, \& D. Psaltis, Sonic-Point Model of Kilohertz Quasi-periodic Brightness Oscillations in Low-Mass X-Ray Binaries, ApJ, 508, 791-830, 1998

Miller, M. C., Evidence for Antipodal Hot Spots During X-Ray Bursts from 4U 1636-536, ApJ, 515, L77-80, 1999

Muno, M. P., D. W. Fox, E. H. Morgan, \& L. Bildsten, Nearly Coherent Oscillations in Type I X-Ray Bursts from KS 1731-260, ApJ, 542, 1016-1033, 2000

Nath, N., T. E. Strohmayer, \& J. H. Swank, Bounds on Compactness for LMXB Neutron Stars from X-ray Burst Oscillations, AAS/High Energy Astrophysics Division, 32, 2305, 2000

Nozakura, T., S. Ikeuchi, \& M. Y. Fujimoto, Lateral Propagation of the Helium Shell Flash on an Accreting Neutron Star, ApJ, 286, 221-231, 1984

Smith, D. A., E. H. Morgan, \& H. V. Bradt, Rossi X-Ray Timing Explorer Discovery of Coherent Millisecond Pulsations during an X-Ray Burst from KS 1731-260, ApJ, 479, L137-L140, 1997

Strohmayer, T. E., W. Zhang, J. H. Swank, A. P. Smale, L. Titarchuk, et al., Millisecond X-Ray Variability from an Accreting Neutron Star System, ApJ, 469, L9-L12, 1996

Strohmayer, T. E., W. Zhang, \& J. H. Swank, 363 HZ Oscillations during the Rising Phase of Bursts from $4 \mathrm{U}$ 1728-34: Evidence for Rotational Modulation, ApJ, 487, L77-L80, 1997

Strohmayer, T. E., K. Jahoda, A. B. Giles, \& U. Lee, Millisecond Pulsations from a Low-Mass X-Ray Binary in the Galactic Center Region, ApJ, 486, 355-362, 1997

Strohmayer, T. E., W. Zhang, J. H. Swank, N. E. White, \& I. Lapidus, On the Amplitude of Burst Oscillations in 4U 1636-54: Evidence for Nuclear-powered Pulsars, ApJ, 498, L135-L138, 1998a

Strohmayer, T. E., W. Zhang, J. H. Swank, \& I. Lapidus, The Long-Term Stability of Oscillations during Thermonuclear X-Ray Bursts: Constraining the Binary X-Ray Mass Function, ApJ, 503, L147-L150, $1998 \mathrm{~b}$

Strohmayer, T. E., Spin-down of Pulsations in the Cooling Tail of an X-Ray Burst from 4U 1636-53, ApJ, 523, L51-L54, 1999

Strohmayer, T. E. \& C. B. Markwardt, On the Frequency Evolution of X-Ray Brightness Oscillations during Thermonuclear X-Ray Bursts: Evidence of Coherent Oscillations, ApJ, 516, L81-L85, 1999

Taylor, J. H., R. N. Manchester, \& A. G. Lyne, Catalog of 558 Pulsars, ApJS, 88, 529-568, 1993

van Straaten, S., M. van der Klis, E. Kuulkers, \& M. Mendez, An Atlas of Burst Oscillations and Spectral Properties in 4U 1728-34, ApJ, submitted, astro-ph/0009194), 2000

Webbink, R. F., S. A. Rappaport, \& G. J. Savonije, On the Evolutionary Status of Bright, Low-mass X-ray Sources, ApJ, 270, 678-693, 1983

Wijnands, R., \& M. van der Klis, Discovery of Two Simultaneous Kilohertz Quasi-periodic Oscillations in 
KS 1731-260, ApJ, 482, L65-L68, 1997

Wijnands, R. \& van der Klis, M. A Millisecond Pulsar in an X-ray Binary System, Nature, 394, 344-346, 1998

Wijnands, R., T. E. Strohmayer, \& L. M. Franco, Discovery of Nearly Coherent Oscillations with a Frequency of $567 \mathrm{~Hz}$ During Type-I X-ray Bursts of the X-ray Transient and Eclipsing Binary X 1658-298, ApJ, in press, (astro-ph/0008526), 2000

Zhang, W., I. Lapidus, N. E. White, \& L. Titarchuk, Kilohertz Quasi-periodic Intensity Oscillations from $4 \mathrm{U}$ 1636-536, ApJ, 469, L17-L20, 1996

Zhang, W., K. Jahoda, R. L. Kelley, T. E. Strohmayer, J. H. Swank et al., Millisecond Oscillations in the Persistent and Bursting Flux of Aquila X-1 during an Outburst, ApJ, 495, L9-L12, 1998 